\title{
BMJ Open Validation of revised patient measures of safety: PMOS-30 and PMOS-10
}

\author{
Gemma Louch (D) , ${ }^{1}$ Caroline Reynolds, ${ }^{1}$ Sally Moore, ${ }^{1}$ Claire Marsh, ${ }^{1}$ \\ Jane Heyhoe, ${ }^{1}$ Abigail Albutt, ${ }^{1}$ Rebecca Lawton (D) , ${ }^{1,2}$ on behalf of the Yorkshire \\ Quality and Safety Research Group
}

To cite: Louch G, Reynolds C, Moore S, et al. Validation of revised patient measures of safety: PMOS-30 and PMOS-10. BMJ Open 2019;9:e031355. doi:10.1136/ bmjopen-2019-031355

- Prepublication history for this paper is available online. To view these files, please visit the journal online (http://dx.doi org/10.1136/bmjopen-2019031355).

Received 30 April 2019 Revised 13 September 2019 Accepted 28 0ctober 2019
Check for updates

(C) Author(s) (or their employer(s)) 2019. Re-use permitted under CC BY. Published by BMJ.

${ }^{1}$ Yorkshire Quality and Safety Research Group, Bradford Institute for Health Research, Bradford, UK

${ }^{2}$ School of Psychology, University of Leeds, Leeds, UK

Correspondence to

Gemma Louch;

Gemma.Louch@bthft.nhs.uk

\begin{abstract}
Objectives There is growing evidence that patients can provide feedback on the safety of their care. The 44-item Patient Measure of Safety (PMOS) was developed for this purpose. While valid and reliable, the length of this questionnaire makes it potentially challenging for routine use. Our study aimed to produce revised, shortened versions of PMOS (PMOS-30 and PMOS-10), which retained the psychometric properties of the longer version. Participants To produce a shortened diagnostic measure, we analysed data from 2002 patients who completed PMOS-44, and examined the reliability of the revised measure (PMOS-30) in a sample of 751 patients. To produce a brief standalone measure, we again analysed data from 2002 patients who completed PMOS-44, and tested the reliability and validity of the brief standalone measure (PMOS-10) in a sample of 165 patients.

Methods The process of shortening the questionnaire involved a combination of secondary data analysis (eg, Standard Deviation and inter-item correlations) and a consensus group exercise to produce PMOS-30 and examine face validity. Analysis of PMOS-30 data examined reliability (eg, Cronbach's alpha). Further secondary data analysis (ie, corrected item-total correlations) produced PMOS-10, and primary data collection assessed its reliability and validity (eg, Cronbach's alpha, analysis of variance).
\end{abstract}

Results Fourteen items were removed to produce PMOS30 and the percentage of negatively worded items was reduced from $57 \%$ to $33 \%$. PMOS-30 demonstrated good internal reliability $(\alpha=0.89)$. The 10 items with the highest corrected item-total correlations across both PMOS-44 and PMOS-30 composed PMOS-10. PMOS-10 had good internal reliability $(\alpha=0.79)$, demonstrated convergent validity; however, discriminant validity was not established. Conclusions Two revised, shortened versions of the original PMOS-44 (PMOS-30 and PMOS-10) were produced to capture patient feedback about safety in hospital. The measures demonstrated good reliability and validity, and preserved the psychometric properties of the original measure.

\section{BACKGROUND}

Measurement has posed an ongoing challenge in patient safety improvement. The difficulties and tensions associated with developing and implementing measures of patient safety are well recognised, and there is a need

\section{Strengths and limitations of this study}

Reliability testing of PMOS-30 took place in a variety of wards across three hospital trusts.

- The involvement of patient representatives is a key strength of the PMOS-10 development phase.

- A limitation of our work is that our studies only included patient participants who could understand English.

for measures to be relevant to multiple stakeholders, have a scientific basis, as well as being feasible and usable. ${ }^{1}$ Indeed, there have been calls for more time and money to be invested in the science of safety measurement and the implementation of systems to capture such measures in order to understand whether safety is improving. ${ }^{2}$ Furthermore, there is recognition that safety cannot be captured in a single measure, and that patients and carers play an essential role in safety monitoring, but are often an underused resource. ${ }^{3}$

The collection of feedback on patient satisfaction and experiences of care is now commonplace, ${ }^{4}$ and in the UK, multiple high profile reports have highlighted the importance of listening and responding to patient views about safety. ${ }^{5-7}$ There is growing evidence that patients can provide feedback on the safety of their care, ${ }^{8}$ and research to suggest that patients can offer a unique perspective on patient safety not captured by other established methods of incident detection. ${ }^{10}$ To provide a proactive diagnostic tool which systematically invites the patient perspective on safety, researchers from the UK developed an intervention to provide a theory and evidence-based approach for the collection of hospital in-patient feedback about safety to support service improvement. The Patient Reporting and Action for a Safe Environment (PRASE) intervention ${ }^{11} 12$ includes two measurement tools. The first is a 44-item theory based measure-the Patient Measure of Safety (PMOS), ${ }^{13} 14$ informed 
by the domains of the Yorkshire Contributory Factors Framework (YCFF) ${ }^{15}$ The second is the Patient Incident Reporting Tool (PIRT) ${ }^{12}$ which enables patients to report safety concerns and positive experiences.

The original measure included a minimum of two items per domain, across nine domains, known to contribute to safety incidents in hospitals: (1) communication and teamworking; (2) organisation and care planning; (3) access to resources; (4) ward type and layout; (5) information flow; (6) staff roles and responsibilities; (7) staff training; (8) equipment (design and functioning) and (9) delays. Within a randomised controlled trial (RCT) of the PRASE intervention, ${ }^{11}$ PMOS data were collected from over 2000 patient participants. The researchers and research nurses who facilitated this data collection reported that some patients, particularly those who were older or very unwell, struggled with the length of the measure and the phrasing of some of the negatively worded items. Evidence suggests that shorter measures and a reduction in assessment time can lessen the burden on participants and improve response rates and missing data. ${ }^{16}$ Therefore, for a large number of questionnaires used in a healthcare context, often short form or brief measures are produced to address this issue with the aim of maintaining acceptable reliability and validity. ${ }^{17} 18$

While in the PRASE RCT ${ }^{11}$ researchers were on hand to answer questions and encourage responses, so the above issues did not pose a major problem (response rates were $86 \%$ ), these issues may result in lower uptake as use becomes more widespread. For example, if data are collected via hospital volunteers (one option that has been tested), difficulties with question wording and/or the length of the measure pose a threat to the reliability and validity of the responses, and may result in missing data. Indeed, in a formative evaluation of the implementation of PRASE with hospital volunteers-volunteers described how the measure was too time consuming for patients to complete, and that its length impacted on the conversation and rapport they were able to build with patients. ${ }^{19}$ Furthermore, in recognition of the potential difficulties described above, researchers in Australia have produced a revised version of the measure for use with vulnerable older adult groups. ${ }^{20}$

\section{OBJECTIVES}

The objectives of this research were to produce two measures that are practical, feasible and simple for hospital inpatients to complete. As it is currently conceived, the PMOS has a diagnostic function. It can be used to identify those factors (see domains above) where attention might most usefully be focused when planning improvement. The first objective therefore was to produce a revised version of PMOS that was shorter with improved acceptability, while at the same time preserving the psychometric properties, conceptual underpinnings and diagnostic function of the longer version. This version would continue to be used, alongside the PIRT tool as a basis for the PRASE intervention. Our second objective was to develop a version of PMOS that was much shorter and that could be used as a brief standalone measure for ongoing monitoring of ward safety performance not focused on planning improvement. Potentially, this measure could be used to prompt additional information gathering effort using the refined diagnostic measure or to monitor safety performance over time.

\section{Patient and public involvement statement}

Patients and the public were involved in this study in the shortening and rewording of the questionnaires. More information is provided in the method.

\section{METHOD}

First, we focused on the diagnostic measure and aimed to reduce the number of PMOS items, rephrase items where appropriate, consider face and content validity, and establish the internal reliability of the revised diagnostic measure. Next we focused on producing a brief standalone measure for on-going monitoring of ward safety performance and establishing its internal reliability, face validity, convergent validity and discriminant validity.

\section{Participants}

\section{PMOS-30}

The shortening exercise included the analysis of PMOS data collected using the original measure (44 items) within the RCT of the PRASE intervention. ${ }^{11}$ Analyses included 2002 patient participants recruited across three hospital trusts and 33 wards in the UK between May 2013 and September 2014. This exercise also included an iterative consensus approach in a series of meetings with researchers from the Yorkshire Quality and Safety Research (YQSR) Group who developed the original measure, and six researchers and research nurses with experience of facilitating the collection of PMOS data in the RCT of the PRASE intervention. These meetings aimed to reduce the number of items in the measure, and considered the phrasing of retained items. At this stage, to establish face validity, we also sought input on item wordings/phrasings from two patient panel representatives (from the YQSR Group's patient panel), and two hospital volunteers involved in a project implementing PRASE in collaboration with hospital volunteers, which we henceforth refer to as the hospital volunteers project ${ }^{192}$

Subsequently, the internal reliability of the revised PMOS was tested in a sample of 751 patients. Patients were recruited by hospital volunteers across 22 wards from three hospitals, at three hospital trusts in the UK between September 2015 and May 2016, within the hospital volunteers project. The characteristics of the patient samples are presented in table 1.

\section{PMOS-10}

The shortening exercise to produce a brief standalone measure took a statistical approach, which involved 
Table 1 Characteristics of the patient samples

\begin{tabular}{|c|c|c|c|c|c|c|}
\hline & \multicolumn{2}{|c|}{$\begin{array}{l}\text { PMOS-44 } \\
\text { N=2002 } \\
\text { Data collected 2013-2014 }\end{array}$} & \multicolumn{2}{|c|}{$\begin{array}{l}\text { PMOS-30 } \\
\text { N=751 } \\
\text { Data collected 2015-2016 }\end{array}$} & \multicolumn{2}{|c|}{$\begin{array}{l}\text { PMOS-10 } \\
\text { N=165 } \\
\text { Data collected 2017-2018 }\end{array}$} \\
\hline Ward type & \multicolumn{2}{|l|}{$\begin{array}{l}\text { Medicine }=41.20 \% \\
\text { Surgery }=56.60 \% \\
\text { Mixed }=2.20 \%\end{array}$} & \multicolumn{2}{|l|}{$\begin{array}{l}\text { Medicine }=41.10 \% \\
\text { Surgery }=49.00 \% \\
\text { Paediatrics }=9.90 \%\end{array}$} & \multicolumn{2}{|c|}{$\begin{array}{l}\text { Medicine }=49.70 \% \\
\text { Surgery }=50.30 \%\end{array}$} \\
\hline Age median, range & \multicolumn{2}{|l|}{65,87} & \multicolumn{2}{|l|}{60,100} & \multicolumn{2}{|l|}{57,71} \\
\hline Gender & \multicolumn{2}{|l|}{$\begin{array}{l}\text { Female }=50.0 \% \\
\text { Male }=50.0 \%\end{array}$} & \multicolumn{2}{|l|}{$\begin{array}{l}\text { Female }=53.5 \% \\
\text { Male }=44.7 \%\end{array}$} & \multicolumn{2}{|l|}{$\begin{array}{l}\text { Female }=55.2 \% \\
\text { Male }=44.8 \%\end{array}$} \\
\hline $\begin{array}{l}\text { Length of stay in days } \\
\text { median, range }\end{array}$ & \multicolumn{2}{|l|}{3,167} & \multicolumn{2}{|l|}{4,90} & \multicolumn{2}{|l|}{3,49} \\
\hline \multirow[t]{13}{*}{ Ethnicity } & African & $0.3 \%$ & African & $0.1 \%$ & African & $1.2 \%$ \\
\hline & Bangladeshi & $0.1 \%$ & Bangladeshi & $0.1 \%$ & Bangladeshi & $0.6 \%$ \\
\hline & British & $93.5 \%$ & British & $89.7 \%$ & Black African & $0.6 \%$ \\
\hline & Caribbean & $0.6 \%$ & Indian & $1.5 \%$ & Indian & $1.2 \%$ \\
\hline & Chinese & $0.2 \%$ & Irish & $0.7 \%$ & Irish & $0.6 \%$ \\
\hline & Indian & $0.5 \%$ & Other & $1.9 \%$ & $\begin{array}{l}\text { Other Asian } \\
\text { Background }\end{array}$ & $0.6 \%$ \\
\hline & Irish & $0.6 \%$ & Other Background & $0.1 \%$ & Pakistani & $7.9 \%$ \\
\hline & Other & $1.2 \%$ & $\begin{array}{l}\text { Other Ethnic } \\
\text { Background }\end{array}$ & $0.5 \%$ & $\begin{array}{l}\text { White \& Black } \\
\text { Caribbean }\end{array}$ & $0.6 \%$ \\
\hline & Other Background & $0.3 \%$ & Pakistani & $4.3 \%$ & White \& Asian & $1.2 \%$ \\
\hline & $\begin{array}{l}\text { Other Mixed } \\
\text { Background }\end{array}$ & $0.2 \%$ & White \& Asian & $0.1 \%$ & White British & $83.6 \%$ \\
\hline & Pakistani & $1.0 \%$ & Missing & $0.9 \%$ & $\begin{array}{l}\text { Other White } \\
\text { Background }\end{array}$ & $1.8 \%$ \\
\hline & $\begin{array}{l}\text { White \& Black } \\
\text { Caribbean }\end{array}$ & $0.2 \%$ & & & & \\
\hline & Missing & $1.1 \%$ & & & & \\
\hline
\end{tabular}

analyses of PMOS data collected using the original measure within the RCT of the PRASE intervention. ${ }^{11}$ The characteristics of the patient samples are presented in table 1.

Prior to data collection, members of the YQSR Group's patient panel reviewed the measure resulting from the statistical exercise to ensure it was acceptable (face validity). The subsequent reliability and validity analyses included 165 patient participants who were recruited across nine wards in one hospital trust in the UK between June 2017 and January 2018. Patient eligibility criteria were those reported in the RCT of the PRASE intervention. ${ }^{11}$

\section{Recruitment and informed consent \\ PMOS-30}

Patients were invited to complete the questionnaire by hospital volunteers within a quality improvement project; therefore, informed consent was not required. The hospital volunteer training package was based on the training for researchers and research nurses who facilitated patient data collection within the RCT of the PRASE intervention. ${ }^{11}$ More information regarding the procedure for hospital volunteers visiting wards has been published previously. ${ }^{19}$

\section{PMOS-10}

Patients were recruited by a research nurse and an undergraduate industrial placement student. Posters were placed on wards, and staff were encouraged to inform patients that researchers were recruiting. Researchers liaised with the nurse in charge to identify patients who had capacity and were considered well enough to take part. Researchers then approached patients to give them information about the study, both written (in the form of a participant information sheet) and verbally. If the patient agreed to take part and gave informed consent, they were recruited into the study.

\section{Measures}

The response options for PMOS-44 (44 items), PMOS-30 (30 items) and PMOS-10 (10 items) are as follows: $1=$ strongly disagree, $2=$ disagree, $3=$ neither agree or disagree, $4=$ agree, $5=$ =strongly agree, not applicable, and I prefer not to answer. Responses are recoded such that a high score indicates more favourable perceptions. 
PMOS-44 and PMOS-30 questionnaires were completed using a laptop/handheld device. PMOS-10 questionnaires were collected on paper. For all versions of the PMOS questionnaire, patients could complete the questionnaire themselves, or completion could be facilitated (researchers/research nurses-PMOS-44; hospital volunteers-PMOS-30; research nurse/industrial placement student-PMOS-10). PMOS-44, PMOS-30, PMOS-10 and 'easy read' versions which have subsequently been produced can be requested via https:/ /www.improvementacademy.org/tools-and-resources/ patient-reportingand-action-for-a-safe-environment.html (freely available).

To examine the convergent validity of PMOS-10, the Friends and Family Test (FFT) ${ }^{22}$ question was asked at the beginning of the PMOS-10 questionnaire. The FFT is a one item measure of patient experience which asks if people would recommend the services they have used and offers a range of responses (extremely likely; likely; neither likely nor unlikely; unlikely; extremely unlikely) - 'How likely are you to recommend this ward to friends and family if they needed similar care or treatment?' A lower score on this measure indicated more favourable perceptions.

\section{Analysis}

All analyses were performed using SPSS V.23. ${ }^{23}$ Our analytical approach was informed by the original PMOS validation study. ${ }^{14}$ For PMOS data, if a patient responded 'not applicable' or 'I prefer not to answer' this was treated as missing data as these responses do not contribute to PMOS scoring. These two additional response options accounted for $87.39 \%$ of missing data points for PMOS44, $72.39 \%$ for PMOS-30 and $99.44 \%$ for PMOS-10. The PMOS-30 and PMOS-10 shortening analyses only included PMOS-44 data where collection was facilitated by a researcher/research nurse, so we could be more confident in the reliability of responses. The PMOS-30 and PMOS-10 reliability and validity analyses included both facilitated and self-completed responses. All missing data were excluded list-wise, the characteristics of patients with missing data were consistent with the larger samples and the retained number of cases differed dependent on the type of analysis performed. There were the following numbers of complete PMOS responses across the data sets: PMOS-44 ( $\mathrm{n}=1119)$; PMOS-30 ( $\mathrm{n}=347)$; PMOS-10 $(\mathrm{n}=119)$, there was no missing data for the FFT question.

\section{Estimate of sample size}

For the PMOS-30 and PMOS-10 reliability and validity analyses, the sample sizes were informed by both statistical and pragmatic considerations, based on a minimum subject to item ratio of at least $10: 1 .^{24} 25$

\section{PMOS-30}

Researchers and research nurses who collected PMOS data from patients within the RCT of the PRASE intervention met to discuss the content of the original measure. Potential items for removal were highlighted, for example, based on patient difficulties in understanding of the item or patient feedback that items were repetitive. Prior to a series of consensus meetings analyses were agreed, the results of which would be brought to the consensus meetings. These analyses included item response variation (Standard Deviation; SD) and inter-item correlations using Pearson's correlation.

Research suggests that for a 5-point scale items which demonstrate reasonable variability (SD of 1.00 or higher) are potential items to retain and that items with limited variance are candidates for removal. ${ }^{26}$ On this basis, items demonstrating low variability (ie, $<0.7$ ) were identified as candidate items for removal, and in terms of the level of overlap between items (possible multicollinearity), high inter-item correlations highlighted candidate items for removal, that is, higher than $0.5 .^{27}$

Subsequently, in a series of consensus meetings with the wider research group, items which had been identified as problematic, based on either researcher/research nurse perceptions or the item analyses, were discussed in greater detail. For the purpose of construct validity, the group considered each potential item for removal, and focused on whether each item reliably tapped what it was intended to measure. For the purpose of content validity, we also endeavoured to ensure a minimum of two items per domain were retained. We used multiple criteria, rather than only a statistical approach when considering items for removal to safeguard against key items being removed.

\section{Reliability analyses}

We examined the internal consistency reliability (Cronbach's alpha) of PMOS-30 data collected from 751 hospital in-patients within the hospital volunteers project. In line with guidance, a reliability coefficient $\alpha$ of $>0.7$ was deemed acceptable. ${ }^{28}{ }^{29}$ For domains with three or more items, we examined the internal consistency reliability in terms of original measure's domain structure, that is, the domains that form the basis of the feedback to support service improvement in the PRASE intervention, and assessed the average inter-item correlations using the recommended values range of $0.15-0.50 .^{30}$ For two item domains, we assessed interitem correlations.

\section{PMOS-10}

Following a method described by Marteau and Bekker, ${ }^{31}$ PMOS items were ranked in order of magnitude of their item-remainder correlations using Pearson's correlation (corrected item-total). PMOS-10 items were selected based on the highest corrected item-total correlations across both PMOS-44 and PMOS-30. PMOS-30 refers to items retained in the 30 -item measure. These analyses were based on the PRASE RCT data; therefore, subsequent rewording and rephrasing of PMOS-30 items were not accounted for. We also examined how PMOS-10 correlated with PMOS-44 and PMOS-30. 
Table 2 Rationale for item removal

Item removed
The following aspects of the ward
made it difficult for staff to do their jobs:

Staff were always able to get advice from other teams about my care if needed

\section{Rationale}

Consensus group discussion

Item analysis

Researchers/research nurses highlighted that frequently patients did Low variability SD $=0.59$ not respond to this item with regard to themselves, and therefore the item was often not answered as intended, that is, how lighting levels affect staff.

Researchers/research nurses highlighted that frequently patients did Low variability SD $=0.67$ not respond to this item in terms of what the item is attempting to tap, that is, specialist staff, etc. High inter-item correlations with 'I got answers to all the questions I had about my care' (0.51)

When staff talked about my care with others, the information they shared was correct

Researchers/research nurses highlighted that frequently patients did Low variability SD $=0.61$ not respond to this item in terms of what the item is attempting to tap that is, information sharing between staff.

The following aspects of the ward $\quad$ Researchers/research nurses highlighted that participants often

Low variability $\mathrm{SD}=0.71$ position of nurses' station

\section{There was equipment that staff found difficult to use (eg, monitoring equipment, beds, hoists)}

Staff were kept waiting for my test results The following aspects of the ward made
it uncomfortable for me: lack of space

Staff always seemed to know what they were meant to be doing
Researchers/research nurses highlighted that frequently patients reported that it would be more appropriate for staff to respond to this item.

Researchers/research nurses felt this issue was covered elsewhere in the measure, that is, 'Information about me that my health care team needed was always available'.

Researchers/research nurses felt that lack of space was assessed elsewhere in the measure, although in relation to staff rather than patients.

'The following aspects of the ward made it difficult for staff to do their jobs: Lack of space'.

\section{Low variability $\mathrm{SD}=0.64$}

Researchers/research nurses highlighted that patients often felt they Low variability SD $=0.59$ could not answer this. Researchers/researcher nurses also felt there was conceptual overlap with another item, that is, 'I got answers to all the questions I had about my care'.

Staff always agreed about my treatment/
care

Nurses were always able to get help from other staff when they asked for it

Researchers/research nurses felt there was conceptual overlap with
another item, that is, 'Staff gave me different information about my care'.

Researchers/research nurses felt there was conceptual overlap with Low variability SD $=0.59$ another item, that is, 'Staff seemed to struggle to get help when they needed it'.

High inter-item correlations with 'The following aspects of the ward made it difficult for staff to do their jobs: Lack of space' $(0.51)$

\section{I knew who to go to if I needed to ask a Researchers/research nurses felt other items capture this issue.}

question

The following aspects of the ward made

it difficult for staff to do their jobs: clutter and untidiness

$\begin{array}{ll}\begin{array}{l}\text { Equipment needed for my care was } \\ \text { always working properly }\end{array} & \begin{array}{l}\text { Researchers/research nurses highlighted that frequently patients } \\ \text { reported that it would be more appropriate for staff to respond to } \\ \text { this item. }\end{array} \\ \begin{array}{ll}\text { The following aspects of the ward made } \\ \text { it uncomfortable for me: other-please } \\ \text { specify }\end{array} & \begin{array}{l}\text { Researchers/research nurses felt this item could be removed due to } \\ \text { low response rate. }\end{array}\end{array}$

SD, Standard Deviation

\section{Validity and reliability analyses}

Taking the same approach as the original PMOS validation study, an overall PMOS-10 'positive index' was constructed by summing the number of items that patients responded to by using one of the two positive response options (strongly agree or agree) for a positively worded item and (strongly disagree or disagree) for a negatively worded item. Therefore, patients could have a score of 10 where higher responses equate to more favourable safety perceptions. If patients had missing responses, the 'positive index' was constructed from the available data.

Internal reliability

We assessed the internal consistency reliability of PMOS-10 (Cronbach's alpha), and in line with guidance, a reliability coefficient $\alpha$ of $>0.7$ was deemed acceptable. ${ }^{28} 29$ 
Table 3 Retained items rephrasing

\section{PMOS-44 item}

Sometimes there was no one available to deal with aspects of my care

Staff did not work together as a team here

The drugs I have been prescribed were always available in the hospital

A doctor changed my plan of care and other staff did not know about it

Staff gave me different information about my care
I have needed treatment and there was no one available who was
trained to do it

Equipment and supplies were not always available when needed (eg, hoists, bed pans, drugs)

I was on a ward that was not able to deal with my treatment needs

After a shift change, staff did not appear to know important

information about my care

It was clear who was in charge of the staff

I always knew which nurse was responsible for my care

On at least one occasion, a member of staff was not able to use the necessary equipment

On at least one occasion, a member of staff was not able to carry out a task that they should have been able to do

My treatment/ procedure/operation did not always happen on time

\section{PMOS-30 item}

There was always someone available to deal with every aspect of my care

Staff worked together as a team here

My medicines were always available

Staff did not always know when a doctor changed my plan of care Staff gave me conflicting information about my care

When I needed treatment, there was always someone available who was trained to do it

Equipment and supplies were always available when needed (eg, hoists, bed pans, walking aids, dressings)

The ward was able to deal with all my treatment needs

After shift changes, staff knew important information about my care

It was clear who was in charge of the ward staff I always knew which nurse or nurses were responsible for my care

Staff were always able to use the necessary equipment

Staff were always able to carry out tasks that they should be able to do

My treatment/procedure/operation always happened on time

\section{Convergent validity}

To determine the convergent validity of the 10-item questionnaire (ie, whether the measure converges with an existing related measure), we examined the association between the PMOS-10 'positive index' and the FFT scores using Pearson's correlation. In line with Cohen's guidelines, ${ }^{32}$ correlations of 0.1 were interpreted as a small effect, 0.3 as medium and 0.5 as large.

\section{Discriminant validity}

A one-way analysis of variance (ANOVA) was conducted to assess the extent to which PMOS-10 discriminated among the nine wards. Ward was entered as a fixed factor, and the PMOS-10 'positive index' as the dependent factor.

\section{RESULTS}

\section{PMOS-30}

Of the 44 items in the original measure, 14 items were removed $(32 \%)$. Table 2 presents the rationale for the removal of each item. Eight items were removed due to both statistical reasons and researcher/research nurse feedback, five items were removed due to researcher/ research nurse feedback alone and one item was removed due to statistical reasons alone. To facilitate the likelihood of reliable and valid responses, we also reduced the quantity of negatively phrased items in the revised measure. In the original measure, the proportion of negatively phrased items was $57 \%$ (25 items); in the revised measure, this was reduced to $33 \%$ (10 items). See table 3 or the rephrasing of retained items.

\section{Internal reliability}

The revised measure (PMOS-30) demonstrated strong reliability, with a coefficient $\alpha$ of 0.89 . Cronbach's alphas and average inter-item correlations were calculated for domains with three or more items, and inter-item correlations (Pearson) were calculated for domains with two items (table 4). Cronbach's alphas for five of the domains ranged from 0.40 to 0.75 , and for these domains, the average inter-item correlations (Pearson) were all within an acceptable range $(0.17-0.38)$, as were the interitem correlations (Pearson) for the two item domains $(0.23-0.42)$.

\section{PMOS-10}

Table 5 shows the 10 highest items (based on corrected item-total correlation) that were included in both PMOS-44 and PMOS-30. These items formed PMOS-10. PMOS-10 correlated with the PMOS-44 ( $\mathrm{r}=0.90, \mathrm{p}=0.000)$ and PMOS-30 ( $\mathrm{r}=0.91, \mathrm{p}=0.000)$.

The mean PMOS-10 'positive index' index score for the entire sample was $8.37(\mathrm{SD}=1.65)$, meaning that on average, patients responded positively to around eight of 


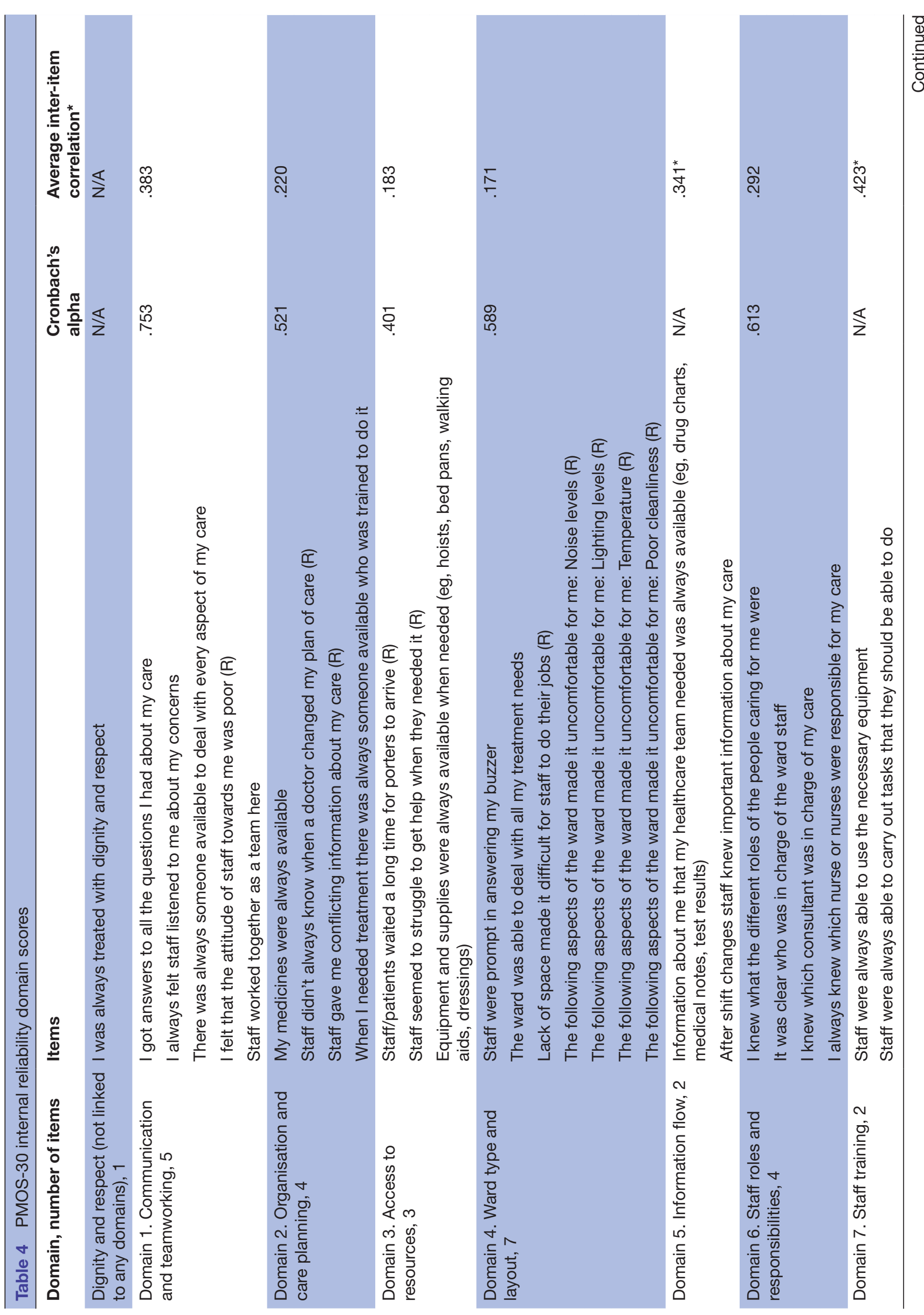


the PMOS-10 items. The recruitment response rate was $92 \%$.

\section{Internal reliability}

The PMOS-10 measure demonstrated good reliability, with a coefficient $\alpha$ of 0.79 .

\section{Convergent validity}

A significant negative correlation was established between the PMOS-10 'positive index' and the FFT scores $(\mathrm{r}=-0.36$, $\mathrm{p}=0.000$ ), indicating that the more positive PMOS scores among patients, the more positive FFT scores were.

\section{Discriminant validity}

A one-way between-groups ANOVA was performed to assess the extent to which the PMOS positive index discriminated among the nine wards. There was no significant main effect of ward $(F(8,156)=1.59, \mathrm{p}=0.130)$.

\section{DISCUSSION}

We aimed to produce two revised patient measures of safety that are practical, feasible and simple for patients to complete, responding directly to the need to enhance patient acceptability of the original measure. ${ }^{1920}$ PMOS-30 included less negatively worded items compared with PMOS-44 (37\% as opposed to 57\%), and the internal reliability of PMOS-30 was established. Reducing the number of negatively worded items addressed the potential for unreliable responses, as previous research suggests that reverse worded items can be contaminated by inattention and confusion. ${ }^{33}$ We retained a minimum of two items per domain across the nine domains known to contribute to safety incidents in hospitals and which formed the basis of the original measure, ${ }^{13} 14$ informed by the YCFF. ${ }^{15}$ In addition, and in collaboration with our patient representatives, items were rephrased to facilitate greater patient understanding. One domain demonstrated an acceptable Cronbach's alpha coefficient and acceptable average inter-item correlation: communication and teamworking. Four domains did not demonstrate an acceptable Cronbach's alpha coefficient but demonstrated an acceptable average inter-item correlation: organisation and care planning, access to resources, ward type and layout and staff roles and responsibilities. Three domains (two item domains) demonstrated acceptable inter-item correlations: information flow, staff training and delays. The reduction in the number of items may have resulted in lowering the reliability statistics of the domains; however, this is to be expected as a reliability coefficient generally increases as the number of items increases. ${ }^{29}$ Interestingly, the domains which performed poorest (eg, ward type and layout; access to resources) also performed poorest in the original PMOS validation study. ${ }^{14}$ Collectively, these findings demonstrate the stability of the original domains over time, even within a shorter, revised measure and suggests that the conceptual underpinnings and psychometric properties of the original measure have been preserved. 
Table 5 Retained items for PMOS-10

\section{Original wording of item in} PMOS-44

Item wording in PMOS-30

I got answers to all the questions I had about my care

had about my care

A doctor changed my plan of care and other staff did not know about it

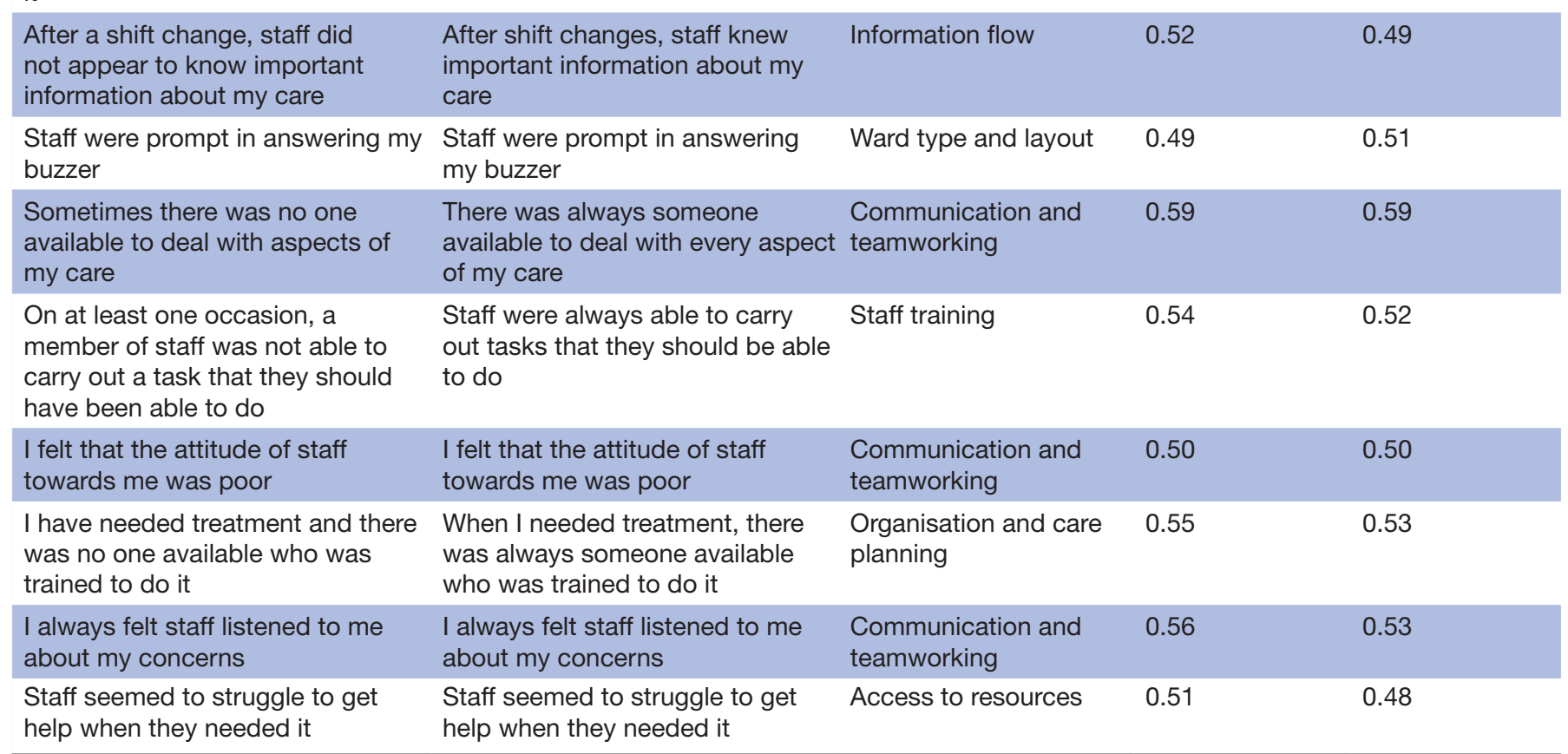

The 10 most highly correlated items that were included in both PMOS-44 (original measure) and PMOS-30 formed the briefer standalone measure (PMOS-10), which was ratified in collaboration with patient representatives to establish face validity. A good alpha coefficient demonstrated the internal reliability of PMOS-10, and convergent validity was established. The strength of the correlation (moderate) suggests that although PMOS-10 'converges' with another known measure of care experience from the patient perspective (FFT), it is indeed measuring something different. To determine discriminant validity, we examined whether PMOS-10 was able to discriminate meaningfully at the ward level, with a view to PMOS-10 being used as a tool to identify those wards which might benefit most from further data gathering efforts (ie, PMOS-30) and improvement as part of the PRASE intervention. There was no significant main effect of ward and no significant differences between wards. Therefore, based on our analyses of nine wards, we cannot purport that PMOS-10 is able to meaningfully discriminate between wards.

There has been substantial research and policy focus on patient involvement in patient safety. ${ }^{34-37}$ Patients providing feedback about the safety of their care is one way for patients to be engaged in patient safety. ${ }^{38}$ The collection of feedback on patient satisfaction and experiences of care are also commonplace. ${ }^{4}$ In terms of safety specifically, there is a wealth of research in support of patients providing feedback about the safety of their care, ${ }^{89}$ and the PRASE intervention, is the first of its kind to collect patient feedback about safety on an on-going basis to support service improvement. Vincent and colleagues' ${ }^{3}$ emphasised that patients and carers play an essential role in safety monitoring, but are often an underused resource. To move from patients and carers being an underused resource in the monitoring of safety, we need to ensure that the measures we do have to capture their perspective are acceptable to patients and carers, are not burdensome to complete and are reliable and valid. The research presented in this paper responds directly to these needs.

\section{Strengths and limitations}

A strength of the development of PMOS-30 is the involvement of six researchers/research nurses with an extensive amount of experience of facilitating PMOS (original measure) data collection, and the involvement of two hospital volunteers and two patient representatives. This provided a rich source of data regarding potential rephrasing of items; leading to considerable face validity. A further strength is that the reliability testing of PMOS-30 took place in a variety of wards across three hospital trusts. The involvement of two patient representatives in the development of PMOS-10 is a key strength. 
A limitation of our work is that our studies only included patient participants who could understand English. Therefore, further testing would be required if PMOS-30 and PMOS-10 were to be translated into other languages. For PMOS-30, some of the domains failing to achieve Cronbach's alpha recommendation of 0.70 is a limitation, even though the average inter-item and inter-item correlations were within the optimum range. For PMOS-10, although the wards recruited were from a variety of medical and surgical specialities, a limitation is the involvement of only one hospital trust. A further limitation is that discriminant validity was not established for PMOS-10. Finally, PMOS-30 had a high amount of missing data which were mainly not applicable responses; therefore, it is possible that a listwise deletion approach may have implications for generalisation.

Implications for health services research, policy and practice By further refining an existing measure, the findings respond to previous research which called for the acceptability of PMOS to be enhanced. The length and some content of the original measure were problematic, and posed issues relating to participant fatigue and burden. The output of a highly reliable and valid shortened diagnostic version of PMOS, which retains the psychometric properties and conceptual underpinnings of the original measure, is integral to the wider adoption and ongoing implementation of the PRASE intervention. The high recruitment response rate $(92 \%)$ for PMOS-10 strengthens the argument that the measure is acceptable to patients, and these findings position PMOS-10 as a reliable and valid brief standalone measure for ongoing monitoring of ward safety performance from the patient perspective. To further validate PMOS-10, research is needed to assess the discriminant validity PMOS-10 on larger scale, in addition to further exploring convergent validity by examining associations with other indicators of patient safety.

\section{CONCLUSION}

Two revised, shortened versions of the original PMOS-44 (PMOS-30 and PMOS-10) were produced to capture patient feedback about safety in hospital, and these measures demonstrated acceptable reliability and validity. PMOS-30 was produced to serve a diagnostic function to be used, alongside the PRASE intervention as a whole. PMOS-10 was developed to be used as a brief standalone measure for ongoing monitoring of ward safety performance.

\section{Twitter Gemma Louch @Gemma_Louch and Rebecca Lawton @LawtonRebecca}

Acknowledgements We would like to thank the Yorkshire Quality and Safety Research (YQSR) Group patient panel for reviewing the PMOS-10 measure, and we would like to thank the two members of the panel and two hospital volunteers for their time and input into revising the PMOS-30 measure. We would also like to thank the patient participants and the staff on the wards for their contribution to the research.

Collaborators Yorkshire Quality and Safety Research Group

Contributors GL led on the development of PMOS-30, carried out the related analyses and drafted the manuscript. JH carried out the PMOS-10 shortening statistical analyses. CR led on the PMOS-10 data collection, and GL, AA and CR carried out the related analyses. SM and CM supported the studies at all stages, in particular the patient and public involvement and engagement element of this work. $\mathrm{RL}$ provided guidance and input at all stages. All authors provided comments and approved the final version.

Funding The research was supported by The Health Foundation, the National Institute for Health Research (NIHR) Yorkshire and Humber Patient Safety Translational Research Centre (NIHR YH PSTRC) and NIHR CLAHRC Yorkshire and Humber www.clahrc-yh.nihr.ac.uk. The PMOS-30 and PMOS-10 shortening exercises utilised data from a wider study funded by the NIHR under its programme Grants for Applied Research scheme ('Improving patient safety through the involvement of patients', RP-PG-0108-10049). The views expressed in this article are those of the author(s) and not necessarily those of The Health Foundation, the NHS, the NIHR or the Department of Health and Social Care.

Competing interests None declared.

Patient consent for publication Not required.

Ethics approval Ethical approvals were obtained from the University of Bradford, Humanities, Social and Health Sciences Research Ethics Panel, and from the East Midlands - Leicester Central Research Ethics Committee.

Provenance and peer review Not commissioned; externally peer reviewed.

Data availability statement The data that support the findings of this study are available from the corresponding author, GL, upon reasonable request. PMOS-44, PMOS-30, PMOS-10 and 'easy read' versions which have subsequently been produced can be requested via https://www.improvementacademy.org/toolsand-resources/patient-reporting-and-action-for-a-safe-environment.html (freely available).

Open access This is an open access article distributed in accordance with the Creative Commons Attribution 4.0 Unported (CC BY 4.0) license, which permits others to copy, redistribute, remix, transform and build upon this work for any purpose, provided the original work is properly cited, a link to the licence is given, and indication of whether changes were made. See: https://creativecommons.org/ licenses/by/4.0\%.

\section{ORCID iDs}

Gemma Louch http://orcid.org/0000-0001-6946-3693

Rebecca Lawton http://orcid.org/0000-0002-5832-402X

\section{REFERENCES}

1 Pronovost PJ, Goeschel CA, Marsteller JA, et al. Framework for patient safety research and improvement. Circulation 2009;119:330-7.

2 Pronovost PJ, Wachter RM. Progress in patient safety: a glass fuller than it seems. Am J Med Qual 2014;29:165-9.

3 Vincent C, Burnett S, Carthey J. The measurement and monitoring of safety: drawing together academic evidence and practical experience to produce a framework for safety measurement and monitoring. The health Foundation 2013.

4 Coulter A, Fitzpatrick R, Cornwell J. Measures of patients' experience in hospital: purpose, methods and uses. London: King's Fund, 2009.

5 Francis R. Report of the mid Staffordshire NHS Foundation trust public inquiry: Executive summary. The Stationery Office, 2013.

6 Keogh B. Review into the quality of care and treatment provided by 14 Hospital trusts in England: overview report, 2013.

7 National Advisory Group on the Safety of Patients in England. A promise to learn a commitment to act: improving the safety of patients in England. National Advisory Group on the safety of patients in England, 2013.

8 O'Hara JK, Armitage G, Reynolds C, et al. How might health services capture patient-reported safety concerns in a hospital setting? an exploratory pilot study of three mechanisms. BMJ Qual Saf 2016.

9 Ward JK, Armitage G. Can patients report patient safety incidents in a hospital setting? A systematic review. BMJ Qual Saf 2012;21:685-99.

10 Armitage G, Moore S, Reynolds C, et al. Patient-Reported safety incidents as a new source of patient safety data: an exploratory comparative study in an acute hospital in England. $J$ Health Serv Res Policy 2018;23:36-43.

11 Lawton R, O'Hara JK, Sheard L, et al. Can patient involvement improve patient safety? a cluster randomised control trial of the patient reporting and action for a safe environment (PRASE) intervention. BMJ Qual Saf 2017;26:622-31.

12 O'Hara JK, Lawton RJ, Armitage G, et al. The patient reporting and action for a safe environment (PRASE) intervention: a feasibility study. BMC Health Serv Res 2016;16:676. 
13 Giles SJ, Lawton RJ, Din I, et al. Developing a patient measure of safety (PMOS). BMJ Qual Saf 2013;22:554-62.

14 McEachan RRC, Lawton RJ, O'Hara JK, et al. Developing a reliable and valid patient measure of safety in hospitals (PMOS): a validation study. BMJ Qual Saf 2014;23:565-73.

15 Lawton R, McEachan RRC, Giles SJ, et al. Development of an evidence-based framework of factors contributing to patient safety incidents in hospital settings: a systematic review. BMJ Qual Saf 2012;21:369-80.

16 Rolstad S, Adler J, Rydén A. Response burden and questionnaire length: is shorter better? A review and meta-analysis. Value in Health 2011;14:1101-8.

17 Kelly L, Sizmur S, Käsbauer S, et al. The relational aspects of care questionnaire: item reduction and scoring using inpatient and accident and emergency data in England. Patient Relat Outcome Meas 2018;9:173-81.

18 Nielsen MB, Hystad SW, Eid J. The brief Norwegian safety climate inventory (brief NORSCl) - psychometric properties and relationships with shift work, sleep, and health. Saf $\mathrm{Sci}$ 2016;83:23-30.

19 Louch G, O'Hara J, Mohammed MA. A qualitative formative evaluation of a patient-centred patient safety intervention delivered in collaboration with Hospital volunteers. Health Expectations 2017;20:1143-53.

20 Taylor N, Hogden E, Clay-Williams R, et al. Older, vulnerable patient view: a pilot and feasibility study of the patient measure of safety (PMOS) with patients in Australia. BMJ Open 2016;6:e011069.

21 Louch G, Mohammed MA, Hughes L, et al. "Change is what can actually make the tough times better": A patient-centred patient safety intervention delivered in collaboration with hospital volunteers. Health Expectations 2019;22:102-13.

22 Department of Health. The NHS friends and family test implementation guidance. Available: https://www.gov.uk/ government/uploads/system/uploads/attachment data/file/213047/ NHS-Friends-and-Family-Test-Implementation-Guidance-v2.pdf [Accessed April 2019].

23 IBM Corp. Ibm SPSS statistics: version 232016.
24 Hatcher L. A step-by-step approach to using the SAS® system for factor analysis and structural equation modeling. Cary, N.C.: SAS Institutte, Inc, 1994.

25 Kyriazos TA. Applied psychometrics: sample size and sample power considerations in factor analysis (EFA, CFA) and SEM in general. Psychology 2018;09:2207-30.

26 Guest G, Namey E. Public health research methods. 392. Thousand Oaks, CA: Sage Publications, 2014.

27 Briggs SR, Cheek JM. The role of factor analysis in the development and evaluation of personality scales. J Pers 1986;54:106-48.

28 Bland JM, Altman DG. Statistics notes: Cronbach's alpha. BMJ 1997;314:572.

29 Tavakol M, Dennick R. Making sense of Cronbach's alpha. Int J Med Educ 2011;2:53-5.

30 Clark LA, Watson D. Constructing validity: basic issues in objective scale development. Psychol Assess 1995;7:309-19.

31 Marteau TM, Bekker H. The development of a six-item short-form of the state scale of the Spielberger State-Trait anxiety inventory (STAI). Br J Clin Psychol 1992;31:301-6.

32 Cohen J. A power primer. Psychol Bull 1992;112:155-9.

33 Sonderen Evan, Sanderman R, Coyne JC. Ineffectiveness of Reverse Wording of Questionnaire Items: Let's Learn from Cows in the Rain. PLoS One 2013:8:e68967.

34 Davis RE, Sevdalis N, Vincent CA. Patient involvement in patient safety: how willing are patients to participate? BMJ Qual Saf 2011;20:108-14.

35 Vincent CA, Coulter A. Patient safety: what about the patient? BMJ Quality Safety 2002;11:76-80.

36 O'Hara J, Lawton R. At a crossroads? key challenges and future opportunities for patient involvement in safety. BMJ Quality \& Safety 2016;0:1-4

37 World Health Organization. Patients for patient safety partnerships for safer health care. Geneva: World Health Organization WHO, 2013. http://www.who.int/patientsafety/patients_for_patient/PFPS_ brochure_2013.pdf

38 The Health Foundation. Evidence scan: involving patients in improving safety. London: The Health Foundation, 2013. 